\title{
A Prognostic Nomogram and Risk Stratification in Pediatric With Hemisphere Lower-Grade Gliomas: a SEER-Based Study 1998-2016
}

\section{Wenqiang Che}

Jinan University First Affiliated Hospital

\section{Fengshuo Xu}

The First Affiliated Hospital of Jinan University

\section{Tong Xu}

Peking University ShenZhen Hospital

Jun Lyu

The First Affiliated Hospital of Jinan University

Xiangyu Wang ( $\nabla$ wang_xy123@126.com )

Jinan University First Affiliated Hospital https://orcid.org/0000-0001-5103-433X

\section{Research Article}

Keywords: Nomogram, SEER, Lower-grade gliomas, Pediatric

Posted Date: February 23rd, 2021

DOI: https://doi.org/10.21203/rs.3.rs-229690/v1

License: (c) (1) This work is licensed under a Creative Commons Attribution 4.0 International License. Read Full License 


\section{Abstract}

\section{Background}

Pediatric lower-grade gliomas (pLGGs) are a rare intracranial tumor that lacks a prognostic prediction model for clinical application. The present study aimed to construct a novel nomogram to predict overall survival for pLGGs.

\section{Methods}

Based on data from the Surveillance, Epidemiology, and End Results (SEER) database, 746 pediatrics diagnosed with cerebral hemispheres lower-grade gliomas from 1998 to 2016 were enrolled for the research. All patients were randomly divided into training and validation datasets at a ratio of 7:3. The Cox and stepwise regression analysis was used to screen the independent prognosticators for developing the nomogram. The discriminating abilities and calibration of the nomogram were assessed by concordance index (C-index), receiver operating characteristic curves (ROCs), area under the ROCs (AUCs), and calibration curves. The accuracy and net benefits of the nomogram were evaluated by comparing it to the traditional prediction method by the net reclassification improvement (NRI), the integrated discrimination improvement (IDI), and the decision curve analysis (DCA). Finally, we employed risk stratifications for pLGGs.

Results

Five independent predicted indicators were associated with OS rates. The constructed nomogram showed reliable discrimination by the $\mathrm{C}$-indexes of the novel nomogram for OS, which were 0.830 and 0.871 , much higher than that in the traditional prediction method ( 0.749 and 0.728 , respectively). The plotted calibration curves showed good consistency between the prediction survival rates and actual observed survival rates in both the training and validation dataset. IDI, NRI, and DCA showed the nomogram had a comparable clinical application to the traditional prediction method. Kaplan-Meier survival curves showed a significant difference among the three risk classifications.

\section{Conclusion}

In conclusion, we developed a novel prognostic nomogram with improved accuracy, better clinical utility, and a more precise prediction of OS rates for hemisphere pLGGs than the traditional prediction method.

\section{Introduction}

Intracranial tumors are the most frequent and commonly diagnosed cancer in pediatric and are among the major causes of cancer-related death(Pollack, 1994). The distribution, epidemiology, pathology, molecular characteristics, prognosis, and treatment modalities for pediatric intracranial tumors have significant differences compared to adult patients(Collins and Pollack, 2020). Gliomas account for 56\% to $70 \%$ of all pediatric central nervous system tumors(Peris-Bonet and Martínez-García et al., 2006). WHO 
grade II/III gliomas are mainly composed of several different histological types, including grade II (diffuse) and grade III (anaplastic) astrocytoma as well as WHO grade II and III oligodendrogliomas. The 2016 WHO Classification of Tumors of the Central Nervous System introduced the IDH mutations and $1 p / 19 q$ codeletion as molecular distinction and definition of WHO II/III gliomas in addition to the so far established histological diagnosis(Louis and Perry et al., 2016), which indicated that lower-grade gliomas (LGGs) should be studied independently, rather than analyzing low-grade or high-grade gliomas simply. The prognosis of adult LGGs has been investigated in numerous studies(Labussiere and Idbaih et al., 2010; Mair and Geurts et al., 2020; Maynard and Okuchi et al., 2020), but no research could offer an effective prediction model for pediatric LGGs (pLGGs).

Nomogram-based clinical prognostic modeling has been one of the most extensively used statistic methods in cancer-related investigations, and the model is accepted as an effective form for prediction prognosis by clinicians(Hu and Bai et al., 2020; Kim and Kim et al., 2020; Li and Lin et al., 2020; Wu and Zhang et al., 2020). Nomogram, a method characterized by visual and mathematical advantages, facilitates the clinical utility and probability calculation of prognosticators or other variables associated with survival. The Surveillance, epidemiology, and end results (SEER) database was established by the National Cancer Institute, aiming to enhance clinical management and reduce patients' cancer burden (Ma and Sun et al., 2014; Yang and Li et al., 2020). Furthermore, the database can offer clinical reference data of a high quality to tumor-related researchers without exposure to highly sensitive personal information. We tried to maximize resources from the SEER database to develop a nomogram with increasing accuracy and better clinical utility for pLGGs to predict prognosis.

\section{Methods}

\section{Patients selection from the SEER database}

Specific clinicopathological data and prognostic outcome of pLGGs from 1998 to 2016 were abstracted from the SEER database using reference number 14622-Nov2018. All the data required by our study were abstracted from the SEER database, an opening database that is freely accessible to the public. Therefore, the study did not require a statement of local ethics approval. The selection criteria were patients younger than 18years old diagnosed with LGGs located in the cerebral hemispheres. The International Classification of Diseases for Oncology was the criteria used to identify cases of LGGs as follows: oligoastrocytomas (9382/3), diffuse astrocytoma (9400/3), pleomorphic xanthoastrocytoma (9424/3), anaplastic astrocytoma (9401/3), oligodendroglioma, NOS (9450/3), and anaplastic oligodendroglioma (9451/3). Exclusion criteria were receiving radiotherapy before surgery performed and a lack of follow-up. The variables were retrieved from the SEER database for further analysis: age at diagnosis, sex, year at diagnosis, race recode, tumor size/site/laterality, histology, surgery, radiation sequence with surgery, chemotherapy, vital status, and survival months.

\section{Definition of variables}


To determine the optimal cutoff values of age at diagnosis, tumor size, and year at diagnosis, $\mathrm{X}$-tile software 3.6.1 was used, and the result was shown in Figure 5. The histological type of LGGs was identified into six categories according to the 2016 WHO World Health Organization classification (WHO): astrocytoma, oligodendroglioma, pleomorphic xanthoastrocytoma, anaplastic astrocytoma, anaplastic oligodendroglioma, undefined. The SEER surgery recorded data on tumor-directed treatments, which were categorized as gross total resection (GTR,), subtotal resection (STR,), receiving surgery with detailed information unknown (RSurgery), or not receiving surgery (NRSurgery). Chemotherapy was classified into two groups: yes and no/unknown. The radiation sequence with surgery was classified into two categories: after surgery and other. Age at diagnosis was classified into three groups: 0-6, 7-16, and 1718 years old. Sex was divided into two groups as male and female. The Year of diagnosis was divided into two intervals: 1998-2011 and 2012-2016. Race/ethnicity was dichotomized as white, black, Asian or Pacific Islander (API), and others. Tumor size was classified into three groups: $\leq 42 \mathrm{~mm},>43 \mathrm{~mm}$, and unknown. Tumor location was divided into four groups: frontal, temporal, parietal, or occipital according to the tumor's primary site. LGGs were located on the left and right side, or others. Moreover, LGGs status was classified into alive and dead.

\section{Statistical analysis}

Firstly, we aimed to establish a comprehensive prognostic nomogram model. All pLGGs patients were divided into training and validation cohorts randomly in a ratio of 7:3. The stepwise regression and multivariate Cox regression analysis were applied for the training cohort to identify inclusion variables in the nomogram. The hazard ratio (HR) and 95\% confidence interval (Cl) were reported. The statistical significance factors that affected overall survival (OS) were included in the prognostic prediction model. OS was the only endpoint of interest in our study and assessed using the Kaplan-Meier curves (Figure 4). The log-rank test was used to compare the difference in survival curves. The nomogram was then constructed to predict 3-, 8- and 8-year survival rates in pLGGs by these identified prognosticators.

Secondly, internal nomogram validation consisted of discrimination, and calibration was performed by using the validation dataset. The concordance index (C-index), the receiver operating characteristic curve (ROC), and the area under the curve (AUC) were calculated by bootstrapping ( $n=500)$ to assess discriminative ability. Calibration curves were plotted to evaluate how predictions from the nomogram model compare to the actual observed outcomes. We applied the net reclassification index (NRI), integrated discrimination improvement (IDI), and decision curve analysis (DCA) to evaluate the prediction accuracy, clinical benefits, and utility of the new prognostic nomogram compared with the traditional prediction method based on histology and surgical treatment. NRI and IDI are two well-established methods for evaluating improvement in risk prediction and measuring the usefulness of a new prediction model. (DCA) was applied to evaluate the potential clinical usefulness and benefits of the predictive nomogram by quantifying net benefits at different threshold probabilities. A two-tail p-value lower than 0.05 was considered to be statistically meaningful. Data analysis was performed with Rstudio based on R programming language and environment (http://www.r-project.org/). 


\section{Results}

\section{Characteristics of pediatric patients and LGGs}

A total of 764 pediatric patients were diagnosed as having LGGs of the cerebral hemispheres and randomly divided into a training and a validation dataset at a ratio of 7:3. The median follow-up was 87 [interquartile range (IQR): 30-150.8] months in all patients, 83 (IQR: 29-147.75) months in the training cohort, and 92.5 (IQR: 39.75-159.5) months in the validation cohort. The demographic and clinical characteristics are shown in Table 1. In the training dataset, the majority of patients were age ranged between 6 and 16 years old (54.79\%), white (81.23\%), male (52.87\%), and diagnosed before 2012 (73.37\%). Moreover, oligodendroglioma was the most common histopathologic type of LGGs, and most of the LGGs were located in the Temporal lobes (44.49\%), on the left side (36.35\%) of whole patients. Of these tumors, there were 341 (44.75\%) tumors with a diameter $\leq 42 \mathrm{~mm}$ and 133 (17.45\%) tumors with a diameter $>43 \mathrm{~mm}$. Besides, most LGGs patients had undergone surgery with detailed information unknown, and gross total resection was performed for 127 patients. Most patients did not receive chemotherapy (75.59\%) and radiotherapy after surgery (79.13\%).

\section{Screening for Prognosticators for pLGGs}

The results of multivariate Cox proportional hazards regression analysis were reported in Figure 1. Surgical status, histological type, tumor size, radiotherapy, and chemotherapy were significantly associated with OS in multivariate analysis and stepwise regression analysis in the training dataset.

\section{Nomogram construction and validation}

A nomogram based on the significant prognostic factors from the training dataset was established to predict LGGs survival probabilities at 3-, 5- and 8-year (Figure 2). The nomogram showed that surgical status was the most significant factor, followed by histopathologic type, radiotherapy, chemotherapy, and tumor size. Each category of every variable corresponds to a score from 0 to 100 on the points scale. The total score was obtained by adding on the individual scores calculated using the nomogram.

The new prediction model's C-index value was 0.830 in the training cohort and 0.871 in the validation cohort, higher than the traditional model, whose C-index value was 0.764 in the training dataset and 0.743 in the validation cohort. Furthermore, the nomogram model's predictive ability was validated by AUCs of ROC curves (Figure 3). In the training dataset, the AUC values were $0.859,0.857$, and 0.848 for 3-, 5- and 8-year OS rates. Moreover, AUC values for 3-, 5- and 8-year survival rates were 0.913, 0.903, and 0.928 in the validation dataset, indicating perfect performance by the nomogram. The plotted calibration curves depicted an excellent agreement between the actual observation of 3,5- and 8-year survival rates and the nomogram's prediction survival rates. In summary, the nomogram for pLGGs had considerable predictive and calibrating abilities.

\section{Clinical value of the nomogram}


Decision curve analysis (DCA), a novel assessment method that analyzed the clinical usability and

benefits of a new prediction model with the traditional model (Figure 6 A-C). For comparison, the new nomograms 3-, 5- and 8-year DCAs exhibited larger net benefits across a range of death risks in the training and validation cohort (Figure 6 D-F). The NRI values in the training and validation dataset for 3year OS were 0.262 (95\% Cl 0.038-0.594) and 0.633 (95\% Cl 0.153-0.997); for 5-year OS were 0.291 (95\% $\mathrm{Cl} 0.070-0.675)$ and 0.539 (95\% Cl 0.112-0.956); for 8-year OS were 0.335 (95\% Cl 0.111-0.670) and 0.779 (95\% $\mathrm{Cl} 0.280-1.350)$, respectively. And the IDI values for 3-, 5-, and 8-year survival rates were $0.052(P \otimes 0.001), 0.058$ ( $P \otimes 0.001)$, and 0.068 ( $P \otimes 0.001)$ in training dataset; 0.089 ( $P \otimes 0.001), 0.901$ ( $P \otimes$ $0.001)$, and $0.105(P \otimes 0.001)$ in validation dataset, respectively. These results suggested that the nomogram predicted prognosis with greater accuracy than the traditional prediction model.

\section{Risk stratification based on the constructed nomogram}

We finally examined further pediatric risk stratification according to total points calculated by the nomogram. Patients with LGGs were divided into three risk classifications: low risk (total points < 103), middle risk (103 $\leq$ total points $<184$ ), and high risk (total points $\geq 184$ ). Kaplan-Meier survival curves for overall survival showed a significant difference among the three risk classifications (Figure 4. F).

\section{Discussion}

In the case of glioma, pediatric and adult patients exhibited apparent epidemiological and

biological characteristics distinctions between and within different WHO grades(Shaw and Wisoff, 2003; Diwanji and Engelman et al., 2017; Zapotocky and Ramaswamy et al., 2018; Jiang and Yang et al., 2019). Tools for predicting the prognosis of adult low-grade and high-grade glioma have been well developed(Zhao and Chen et al., 2019; Yang and Yao et al., 2020), but there was inadequate clinical evidence on the prognosis of pediatric LGGs. Therefore, we developed a prognostic nomogram in the current study to predict pediatric patients' prognosis with LGGs using the data derived from the SEER database. The nomogram provides refined prognostication ability compared with the traditional prediction method based on surgical status and histology(Chen and Smith-Cohn et al., 2017). The calibration curves indicated that the predicted 3-, 5-, and 8-year OS rates closely corresponded to the actual observed survival rates regarding both the training and validation cohorts. Five covariables were screened and incorporated into the nomogram. According to standard deviation along with nomogram scales, surgery was the most important prognosticator, followed by histology, radiotherapy, chemotherapy, and tumor size.

Some prognostic variables potentially affect the outcome of pediatric patients with glioma, such as age at diagnosis and tumor site. These two factors were fully taken into consideration by us in the present research. Costa et al. considered that the OS significantly correlated the age of pediatric patients(Silva Da Costa and Camargo et al., 2020). Another large retrospective population-based cohort study proposed that age younger than three years predicted a greater likelihood of survival in pediatric with high-grade 
gliomas and a poor survival with low-grade glioma(Qaddoumi and Sultan et al., 2009). In our study, age was not associated with prognosis. In contrast, a previous study found age was not associated with survival pediatric cohorts(Mistry and Mummareddy et al., 2020), which was the same as our study results. The small number of patients and inappropriate grouping could conceivably contribute to the contradictory result. Tumor site was another controversial factor. Frontal glioma location has been associated with a better prognosis in adult LGGs for the preferential distribution of IDH mutated(Gorlia and Delattre et al., 2013; Gorlia and Wu et al., 2013; Delfanti and Piccioni et al., 2017). However, the situation is different in pediatric LGGs. As we can see, the tumor site was not associated with prognosis in our study. Patients with diffuse midline gliomas (DMGs) have dismal outcomes(Mueller and Taitt et al., 2020). Furthermore, DMGs are mainly located in the brainstem in pediatric patients(Mackay and Burford et al., 2017; Mueller and Jain et al., 2019). Therefore, the result was acceptable when midline and brainstem gliomas were not involved in our study.

As a first-line treatment, surgical treatment, especially maximal safe resection, is often desired, whenever feasible in pediatric high-grade gliomas(Coleman and Stoller et al., 2020) and low-grade glioma(Goebel and Gnekow et al., 2019). A series of research indicated significant correlations between the increased extent of resection and OS and progression-free survival(Pogorzala and Styczynski et al., 2014; Walston and Hamstra et al., 2015; Adams and Adams et al., 2016; Goebel and Gnekow et al., 2019). Surgery was the strongest protection predictor of OS in the present study. The significant difference in survival emphasized the importance of surgery and indicated how surgical resection plays a key role in improving the survival in pLGGs. Although radiotherapy is an important treatment for malignant pediatric central nervous system tumors, the overall benefit and optimal timing of performing the radiotherapy for pHGGs and low-grade gliomas are not clear(Coleman and Stoller et al., 2020; Collins and Pollack, 2020). The survival advantage of radiotherapy was not seen in pediatric patients given a total or near-total tumor resection(Hanzély and Polgár et al., 2003), and poor OS was associated with the nonirradiated group(Pollack and Claassen et al., 1995). Similarly, the results of our study do not support radiation for pLGGs. Moreover, our study's data did not demonstrate any benefit of chemotherapy in terms of overall survival. Until now, no standard chemotherapy strategies widely accepted have been introduced into the child neuro-oncology community(Coleman and Stoller et al., 2020). Nuanced and systematic research is still required to optimize selection criteria for radiotherapy and chemotherapy; however, there appear to be suitable candidates for some specific radiotherapy and chemotherapy paradigms.

Histological criteria were the second strongest independent predictor of prognosis in the study. Tumor grade was a good predictor of survival, and both previous and the present found the association between WHO grade II gliomas and better OS(Qaddoumi and Sultan et al., 2009). Different immune infiltration types may contribute to the difference in prognosis between Oligodendroglioma and astrocytoma(Song and Weng et al., 2020). Pleomorphic xanthoastrocytoma (PXA) is a rare brain tumor mainly affecting pediatric and young adults(Vaubel and Zschernack et al., 2020). PXA with anaplastic features usually confers a less favorable outcome though uncommon at initial manifestation. Recent research has indicated that tumor size was another significant prognosticator for pediatric gliomas, and small tumor size was regarded as a prognostic protection factor(Goebel and Gnekow et al., 2019; Collins and Pollack, 
2020). The exact value of tumor size is, however, currently unclear. In contrast, the present study carefully analyzed the association between tumor size and OS, and the value was determined as $42 \mathrm{~mm}$ eventually.

The traditional prognosis prediction method based on histologic prognostic factors and surgery cannot meet the clinical needs due to the low accuracy(Chen and Smith-Cohn et al., 2017). Survival may vary from months to multiple years for patients with the same diagnosis, which could not be explained simply by the traditional method. Therefore, we take the opportunity to reconsider more factors that could be enrolled into the prognostic nomogram, including radiotherapy, chemotherapy, and tumor size. We further compared the novel nomogram, which involves five variables, with the traditional prognostic method based on only two variables. According to the nomogram's IDI and NRI values, the present study showed that the new model had a better predictive capability. Moreover, we used DCAs to verify the nomogram's predictive power with better benefits and clinical utility than the traditional method.

Based on the nomogram scores, all pediatrics were divided into three groups as follows: low-risk, middlerisk and high-risk. Kaplan-Meier survival analysis shows that patients with different risk levels have a significantly different OS. Particularly, the nomogram can provide more clinical information for discriminating patients at high risk. Identifying the pLGGs at a high risk of death is critical for disease management and decreased mortality. Accordingly, the pLGGs with total scores larger than 182 should receive extra attention.

The nomogram has exhibited excellent clinical application value and potential value. We first analyzed a large dataset of supratentorial pLGGs through the SEER database, a large population retrospective database representing the $28 \%$ populations of different US states. When calculating C-index, AUC values, and calibration curves, Transparent reporting of a multivariable prediction model for individual prognosis or diagnosis (TRIPOD)(Collins and Reitsma et al., 2015) was the criteria that we abided by.

This study has some limitations. First, it was a retrospective analysis that is never able to prove causality. Secondly, the database could not offer details information on radiotherapy and chemotherapy. Thirdly, information about gene mutations and chromosomal deletions was unavailable from the database.

\section{Conclusions}

In conclusion, we developed a novel prognostic nomogram with improved accuracy, better clinical utility, and more precise prediction of OS rates for hemisphere PLGGs than the traditional prediction method.

\section{Declarations}

Funding: This study received no external funding.

Conflicts of Interest: The authors declare that no conflict of interest exists. 
Availability of data and material: All data needed to assess the conclusions in the paper are present in the paper.

Code availability: Not applicable.

Ethics approval: "Not applicable." For the study based on the SEER database, an opening database freely accessible to the public.

Consent to participate: Not applicable.

Consent for publication: All necessary publication consent was obtained prior to research.

Author Contributions: Methodology, C.W.; software, C.W.; validation, X.F, L.J. and W.X.; formal analysis, C.W. and X.T.; resources, C.W., X.F.; writing-original draft preparation, C.W.; writing-review and editing, W.X., L.J.; supervision, W.X., L.J.; project administration, W.X. All authors have read and agreed to the published version of the manuscript.

\section{References}

Adams, H. and H. H. Adams, et al. (2016). "Evaluating extent of resection in pediatric glioblastoma: a multiple propensity score-adjusted population-based analysis." Child's Nervous System 32 (3): 493-503.

Chen, R. and M. Smith-Cohn, et al. (2017). "Glioma subclassifications and their clinical significance." Neurotherapeutics 14 (2): 284-297.

Chen, R. and M. Smith-Cohn, et al. (2017). "Glioma subclassifications and their clinical significance." Neurotherapeutics 14 (2): 284-297.

Coleman, C. and S. Stoller, et al. (2020). "Pediatric hemispheric high-grade glioma: Targeting the future." Cancer and Metastasis Reviews 39 (1): 245-260.

Coleman, C. and S. Stoller, et al. (2020). "Pediatric hemispheric high-grade glioma: Targeting the future." Cancer and Metastasis Reviews 39 (1): 245-260.

Collins, G. S. and J. B. Reitsma, et al. (2015). "Transparent reporting of a multivariable prediction model for individual prognosis or diagnosis (TRIPOD) the TRIPOD statement." Circulation 131 (2): 211-219.

Collins, K. L. and I. F. Pollack (2020). "Pediatric Low-Grade Gliomas." Cancers 12 (5).

Delfanti, R. L. and D. E. Piccioni, et al. (2017). "Imaging correlates for the 2016 update on WHO classification of grade II/III gliomas: implications for IDH, 1p/19q and ATRX status." Journal of neurooncology 135 (3): 601-609.

Diwanji, T. P. and A. Engelman, et al. (2017). "Epidemiology, diagnosis, and optimal management of glioma in adolescents and young adults." Adolescent health, medicine and therapeutics 8: 99-113. 
Goebel, A. M. and A. K. Gnekow, et al. (2019). "Natural History of Pediatric Low-Grade Glioma Disease First Multi-State Model Analysis." Journal of Cancer 10 (25): 6314-6326.

Gorlia, T. and J. Delattre, et al. (2013). "New clinical, pathological and molecular prognostic models and calculators in patients with locally diagnosed anaplastic oligodendroglioma or oligoastrocytoma. A prognostic factor analysis of European Organisation for Research and Treatment of Cancer Brain Tumour Group Study 26951." European journal of cancer 49 (16): 3477-3485.

Gorlia, T. and W. Wu, et al. (2013). "New validated prognostic models and prognostic calculators in patients with low-grade gliomas diagnosed by central pathology review: a pooled analysis of EORTC/RTOG/NCCTG phase III clinical trials." Neuro-oncology 15 (11): 1568-1579.

Hanzély, Z. and C. Polgár, et al. (2003). "Role of early radiotherapy in the treatment of supratentorial WHO Grade II astrocytomas: long-term results of 97 patients." Journal of neuro-oncology 63 (3): 305-312.

$\mathrm{Hu}, \mathrm{P}$. and J. Bai, et al. (2020). "Trends of incidence and prognosis of gastric neuroendocrine neoplasms: a study based on SEER and our multicenter research." Gastric cancer : official journal of the International Gastric Cancer Association and the Japanese Gastric Cancer Association 23 (4): 591-599.

Jiang, H. and K. Yang, et al. (2019). "Diffuse midline glioma with an H3 K27M mutation: A comparison integrating the clinical, radiological, molecular features between adult and pediatric patients." Neurooncology.

Kim, B. H. and S. Kim, et al. (2020). "Development of an Individualized Prediction Calculator for the Benefit of Postoperative Radiotherapy in Patients with Surgically Resected De Novo Stage IV Breast Cancer." Cancers 12 (8).

Labussiere, M. and A. Idbaih, et al. (2010). "All the 1p19q codeleted gliomas are mutated on IDH1 or IDH2." Neurology 74 (23): 1886-1890.

$\mathrm{Li}, \mathrm{J}$. and Y. Lin, et al. (2020). "Prognostic nomogram based on the metastatic lymph node ratio for gastric neuroendocrine tumour: SEER database analysis." ESMO open $\mathbf{5}$ (2).

Louis, D. N. and A. Perry, et al. (2016). "The 2016 World Health Organization classification of tumors of the central nervous system: a summary." Acta neuropathologica 131 (6): 803-820.

Ma, H. and H. Sun, et al. (2014). "Survival improvement by decade of patients aged 0-14 years with acute lymphoblastic leukemia: a SEER analysis." Scientific reports 4 (1): 1-7.

Mackay, A. and A. Burford, et al. (2017). "Integrated molecular meta-analysis of 1,000 pediatric high-grade and diffuse intrinsic pontine glioma." Cancer cell 32 (4): 520-537. e5.

Mair, M. J. and M. Geurts, et al. (2020). "A basic review on systemic treatment options in WHO grade II-III gliomas." Cancer treatment reviews 92: 102124. 
Maynard, J. and S. Okuchi, et al. (2020). "World Health Organization Grade II/III Glioma Molecular Status: Prediction by MRI Morphologic Features and Apparent Diffusion Coefficient." Radiology 296 (1): 111-121.

Mistry, A. M. and N. Mummareddy, et al. (2020). "Association between supratentorial pediatric high-grade gliomas involved with the subventricular zone and decreased survival: a multi-institutional retrospective study." Journal of neurosurgery. Pediatrics: 1-7.

Mueller, S. and J. M. Taitt, et al. (2020). "Mass cytometry detects H3.3K27M-specific vaccine responses in diffuse midline glioma." The Journal of clinical investigation 130 (12): 6325-6337.

Mueller, S. and P. Jain, et al. (2019). "A pilot precision medicine trial for children with diffuse intrinsic pontine glioma-PNOC003: A report from the Pacific Pediatric Neuro-Oncology Consortium." International journal of cancer 145 (7): 1889-1901.

Peris-Bonet, R. and C. Martínez-García, et al. (2006). "Childhood central nervous system tumoursincidence and survival in Europe (1978-1997): report from Automated Childhood Cancer Information System project." European journal of cancer 42 (13): 2064-2080.

Pogorzala, M. and J. Styczynski, et al. (2014). "Survival and prognostic factors in children with brain tumors: long-term follow-up single center study in Poland." Anticancer research 34 (1): 323-6.

Pollack, I. F. (1994). "Brain tumors in children." New England Journal of Medicine 331 (22): 1500-1507.

Pollack, I. F. and D. Claassen, et al. (1995). "Low-grade gliomas of the cerebral hemispheres in children: an analysis of 71 cases." Journal of neurosurgery 82 (4): 536-547.

Qaddoumi, I. and I. Sultan, et al. (2009). "Outcome and prognostic features in pediatric gliomas: a review of 6212 cases from the Surveillance, Epidemiology, and End Results database." Cancer: Interdisciplinary International Journal of the American Cancer Society 115 (24): 5761-5770.

Qaddoumi, I. and I. Sultan, et al. (2009). "Outcome and prognostic features in pediatric gliomas: a review of 6212 cases from the Surveillance, Epidemiology, and End Results database." Cancer 115 (24): 5761-70.

Shaw, E. G. and J. H. Wisoff (2003). "Prospective clinical trials of intracranial low-grade glioma in adults and children." Neuro-oncology 5 (3): 153-60.

Silva Da Costa, M. D. and N. C. Camargo, et al. (2020). "High-grade gliomas in children and adolescents: is there a role for reoperation?" Journal of neurosurgery. Pediatrics: 1-10.

Song, L. R. and J. C. Weng, et al. (2020). "Prognostic and predictive value of an immune infiltration signature in diffuse lower-grade gliomas." JCl insight 5 (8).

Vaubel, R. and V. Zschernack, et al. (2020). "Biology and grading of pleomorphic xanthoastrocytoma-what have we learned about it?" Brain pathology (Zurich, Switzerland). 
Walston, S. and D. A. Hamstra, et al. (2015). "A multi-institutional experience in pediatric high-grade glioma." Frontiers in oncology 5: 28.

Wu, J. and H. Zhang, et al. (2020). "A nomogram for predicting overall survival in patients with low-grade endometrial stromal sarcoma: A population-based analysis." Cancer communications (London, England) 40 (7): 301-312.

Yang, J. and Y. Li, et al. (2020). "Brief introduction of medical database and data mining technology in big data era." Journal of Evidence-Based Medicine 13 (1): 57-69.

Yang, Y. and M. Yao, et al. (2020). "Prognostic Nomograms for Primary High-Grade Glioma Patients in Adult: A Retrospective Study Based on the SEER Database." BioMed research international 2020.

Zapotocky, M. and V. Ramaswamy, et al. (2018). "Adolescents and young adults with brain tumors in the context of molecular advances in neuro-oncology." Pediatric blood \& cancer 65 (2): e26861.

Zhao, Y. and S. Chen, et al. (2019). "A nomogram for predicting individual prognosis of patients with lowgrade glioma." World neurosurgery 130: e605-e612.

\section{Tables}

Table 1 Demographics and clinicopathological characteristics of Pediatric lower-grade gliomas 


\begin{tabular}{|c|c|c|c|c|c|}
\hline Characteristics & Categories & Total (\%) & $\begin{array}{l}\text { Training } \\
(\%)\end{array}$ & Validation (\%) & $\begin{array}{l}\mathrm{P} \\
\text { Value }\end{array}$ \\
\hline \multirow[t]{3}{*}{ Age } & $0 y-6 y$ & $188(24.67)$ & $130(24.90)$ & $58(25.89)$ & 0.9521 \\
\hline & $7 y-16 y$ & $408(53.54)$ & 286(54.79) & $122(54.46)$ & \\
\hline & $17 y-18 y$ & 150(19.69) & 106(20.31) & $44(19.64)$ & \\
\hline \multirow[t]{2}{*}{ Year } & Before 2012 & $552(72.44)$ & $383(73.37)$ & 169(75.45) & 0.5538 \\
\hline & After 2012 & $194(25.46)$ & 139(26.63) & $55(24.55)$ & \\
\hline \multirow[t]{2}{*}{ Sex } & Male & $391(51.34)$ & $276(52.87)$ & $115(51.34)$ & 0.7005 \\
\hline & Female & $355(46.59)$ & $246(47.13)$ & $109(48.66)$ & \\
\hline \multirow[t]{4}{*}{ Race } & White & $598(78.48)$ & $424(81.23)$ & 174(77.68) & 0.2533 \\
\hline & Black & $86(11.29)$ & $54(10.34)$ & $32(14.29)$ & \\
\hline & API & $42(5.51)$ & $32(6.13)$ & $10(4.46)$ & \\
\hline & Other & $20(2.62)$ & $12(2.30)$ & $8(3.57)$ & \\
\hline Histological & astrocytoma & $246(32.28)$ & $170(32.57)$ & 76(33.93) & 0.1434 \\
\hline \multirow[t]{5}{*}{ type } & oligodendroglioma & $182(23.88)$ & $119(22.80)$ & $63(28.13)$ & \\
\hline & $\begin{array}{l}\text { pleomorphic } \\
\text { xanthoastrocytoma }\end{array}$ & $125(16.40)$ & $84(16.09)$ & $41(18.30)$ & \\
\hline & Ana-astrocytoma & 108(14.17) & $85(16.28)$ & $23(10.27)$ & \\
\hline & Ana- oligodendroglioma & $18(2.36)$ & $12(2.30)$ & $6(2.68)$ & \\
\hline & undefined & $67(8.79)$ & $52(9.96)$ & $15(6.70)$ & \\
\hline \multirow[t]{4}{*}{ Site } & Frontal & $249(32.68)$ & $173(33.14)$ & $76(33.93)$ & 0.5618 \\
\hline & Temporal & $339(44.49)$ & $235(45.02)$ & $104(46.43)$ & \\
\hline & Parietal & $119(15.62)$ & $89(17.05)$ & $30(13.39)$ & \\
\hline & Occipital & $39(5.12)$ & $25(4.79)$ & $14(6.25)$ & \\
\hline \multirow[t]{3}{*}{ Size(mm) } & $\leq 42$ & $341(44.75)$ & $246(47.13)$ & $95(42.41)$ & 0.4937 \\
\hline & $₫ 43$ & $133(17.45)$ & $91(17.43)$ & $42(18.75)$ & \\
\hline & Other & $272(35.70)$ & $185(35.44)$ & $87(38.84)$ & \\
\hline \multirow[t]{3}{*}{ Laterality } & Right & $269(35.30)$ & $190(36.40)$ & $79(35.27)$ & 0.9295 \\
\hline & Left & $277(36.35)$ & 194(37.16) & $83(37.05)$ & \\
\hline & Other & $200(26.25)$ & $138(26.44)$ & $62(27.68)$ & \\
\hline
\end{tabular}




\begin{tabular}{|llllll|} 
Surgery & GTR & $127(16.67)$ & $95(18.20)$ & $32(14.29)$ & 0.1964 \\
& STR & $51(6.69)$ & $30(5.57)$ & $21(9.38)$ & \\
& Receiving surgery & $492(64.57)$ & $342(65.52)$ & $150(66.96)$ & \\
\cline { 2 - 4 } & No surgery & $76(9.97)$ & $55(10.54)$ & $21(9.38)$ & \\
\hline \multirow{2}{*}{ Radiotherapy } & After Surgery & $143(18.77)$ & $103(19.73)$ & $40(17.86)$ & 0.5510 \\
& Other & $603(79.13)$ & $419(80.27)$ & $184(82.14)$ & \\
\hline \multirow{2}{*}{ Chemotherapy } & Yes & $170(22.31)$ & $117(22.41)$ & $53(23.66)$ & 0.7098 \\
& No/Unknown & $576(75.59)$ & $405(77.59)$ & $171(76.34)$ & \\
\hline \multirow{2}{*}{ Months } & & $87(30-$ & $83(29-$ & $92.5(39.75-$ & \\
& & $150.8)$ & $147.75)$ & $159.5)$ & \\
\hline
\end{tabular}

API, Asian or Pacific Islander; GTR, gross total resection; STR. Subtotal resection.

\section{Figures}




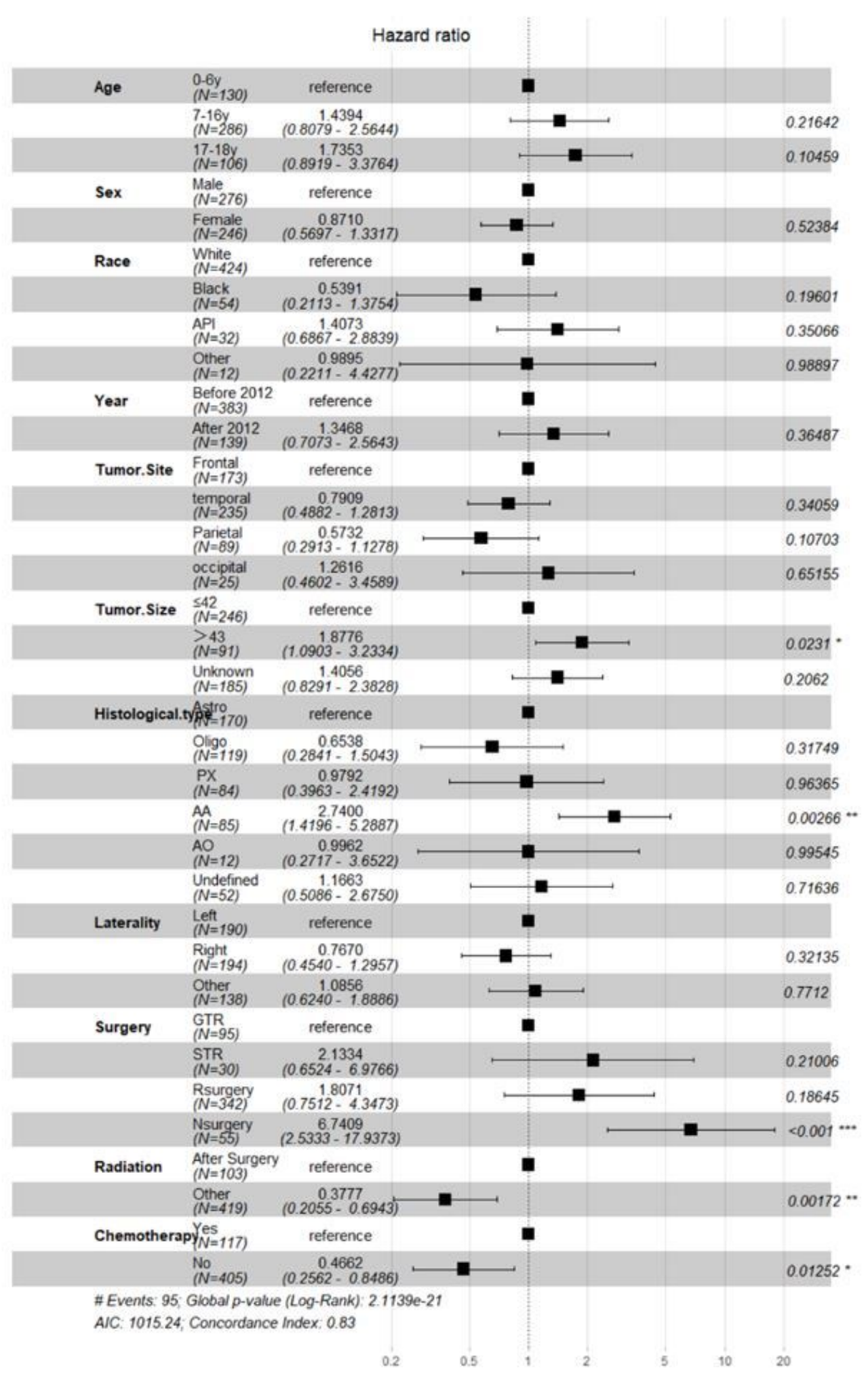

\section{Figure 1}

Multivariate Cox analysis of factors of overall survival. 
Points

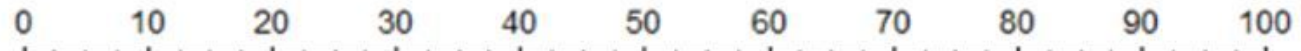

Tumor.Size

Chemotherapy

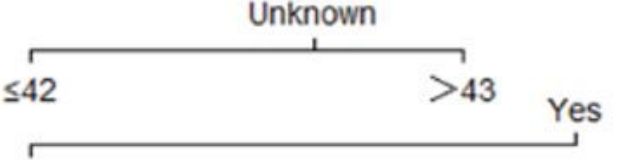

Radiation

Histological.type

Surgery

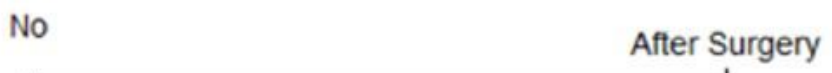

Other Astro AO AA
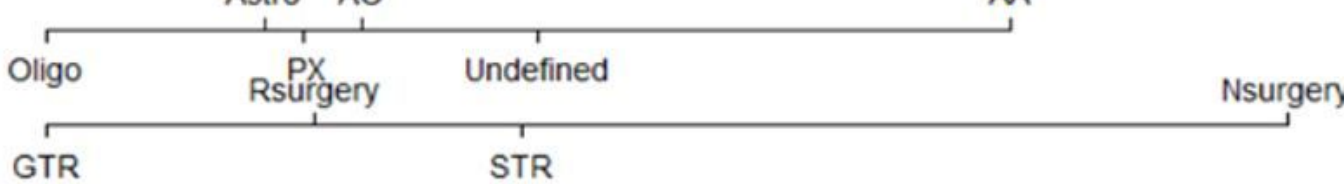

Total Points

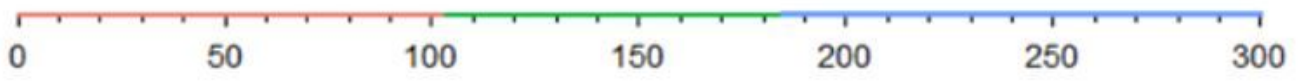

3-years Survival Probability

5-years Survival Probability

8-years Survival Probability

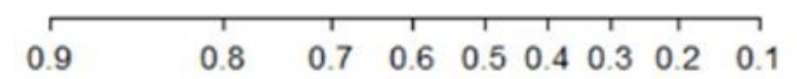

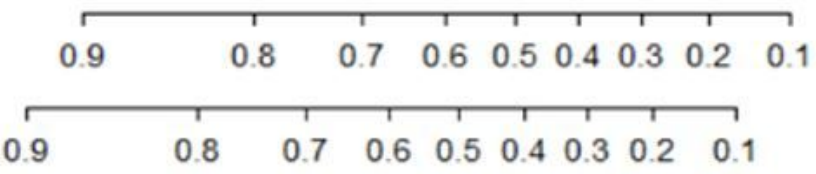

\section{Figure 2}

A developed nomogram for prognostic prediction of pediatric lower-grade gliomas (pLGGs) overall survival rates at 3-, 5-, and 8-year. pLGGs were divided into three risk classifications: low risk (total points $<103$, pink), middle risk (103 $\leq$ total points < 184, green), and high risk (total points $\geq 184$, blue).
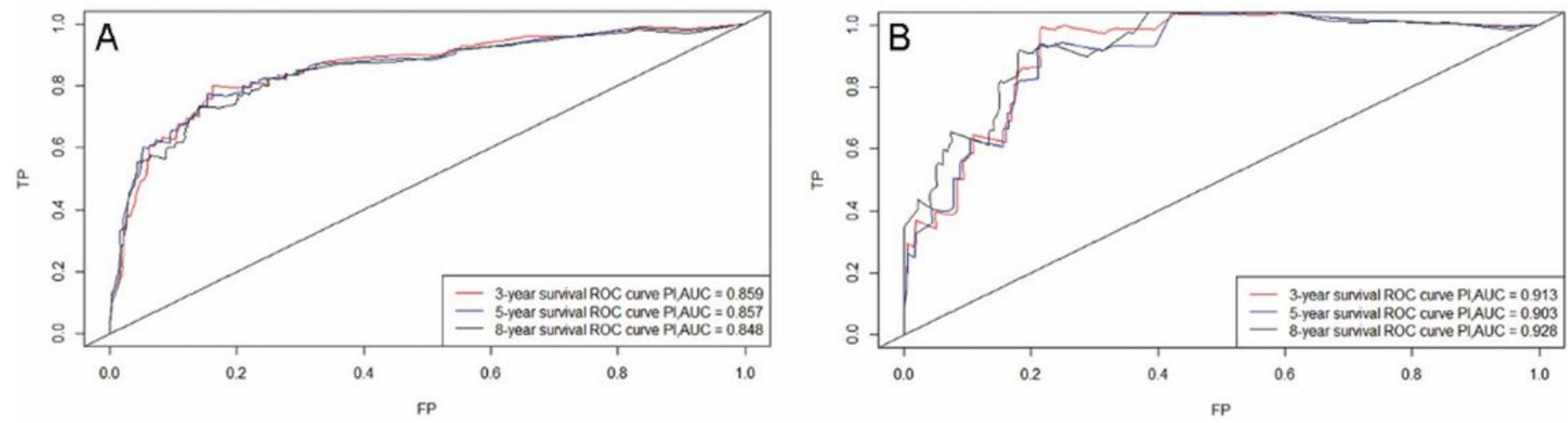

\section{Figure 3}

ROCs curve for nomograms. AUCs of the nomograms to predict overall survival at 3-years, 5-year and 8year using training cohort (A) and validation cohort (B). ROC, receiver operating characteristic; AUCs, area 
under the curves.
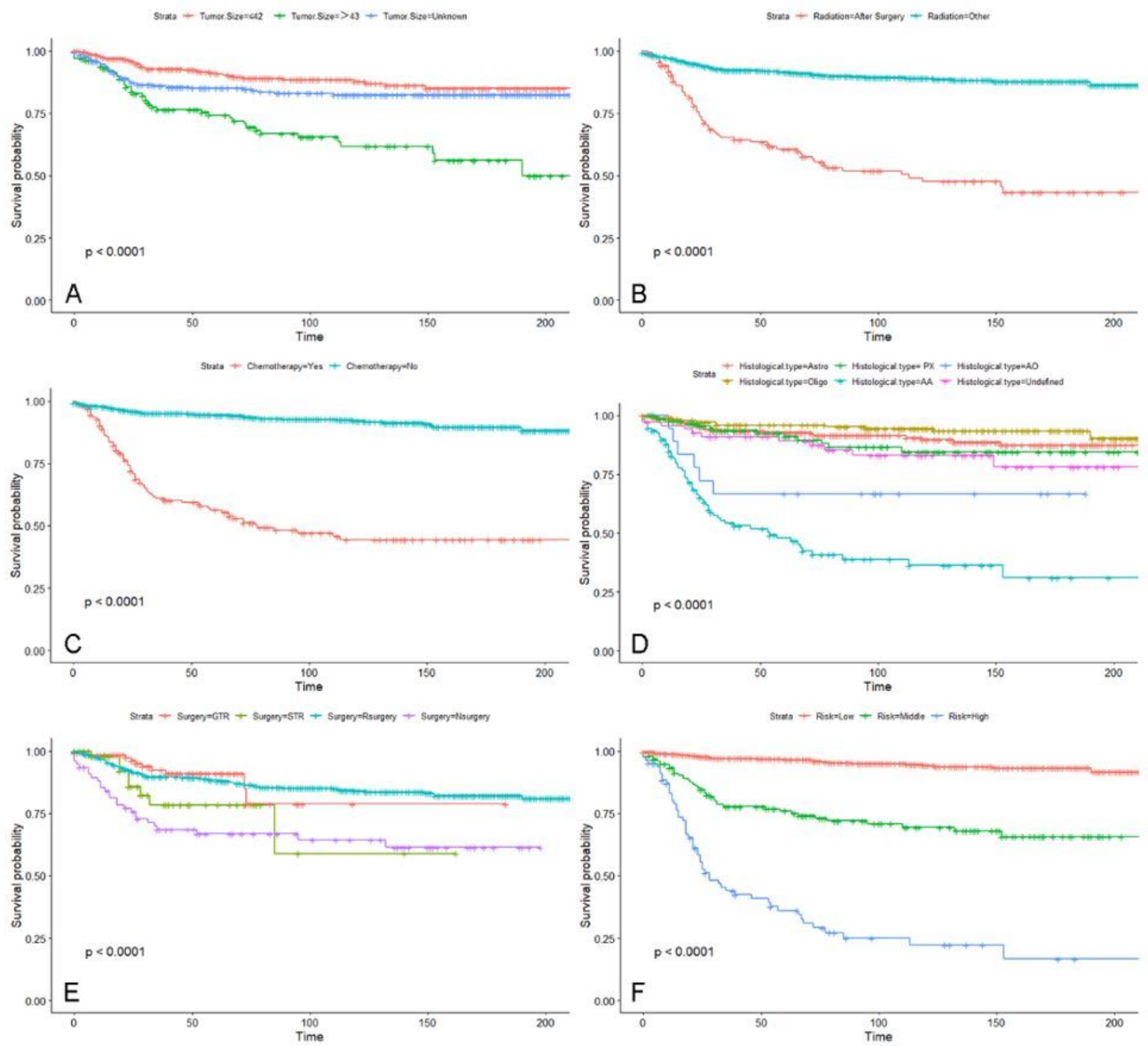

\section{Figure 4}

Kaplan-Meier method used to estimate overall survival of pediatric lower-grade gliomas stratified by tumor size $(A)$, radiation $(B)$, chemotherapy $(C)$, histological type (D), surgery $(E)$, and risk classifications (E). 


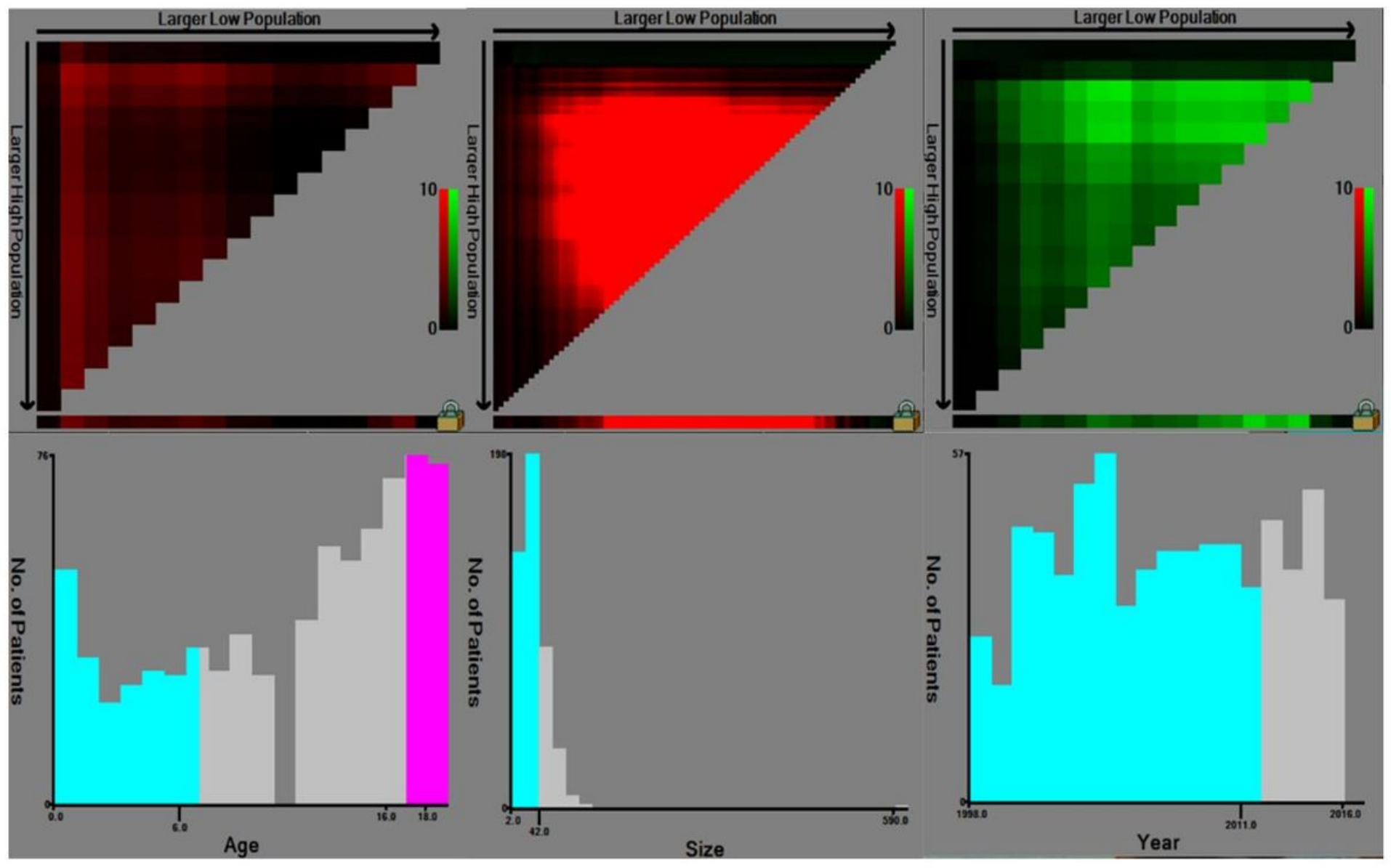

\section{Figure 5}

Graphs show optimal cut-off values of age at diagnosis (Left), tumor size (Middle) and year at diagnosis (Right) via X-tile software.
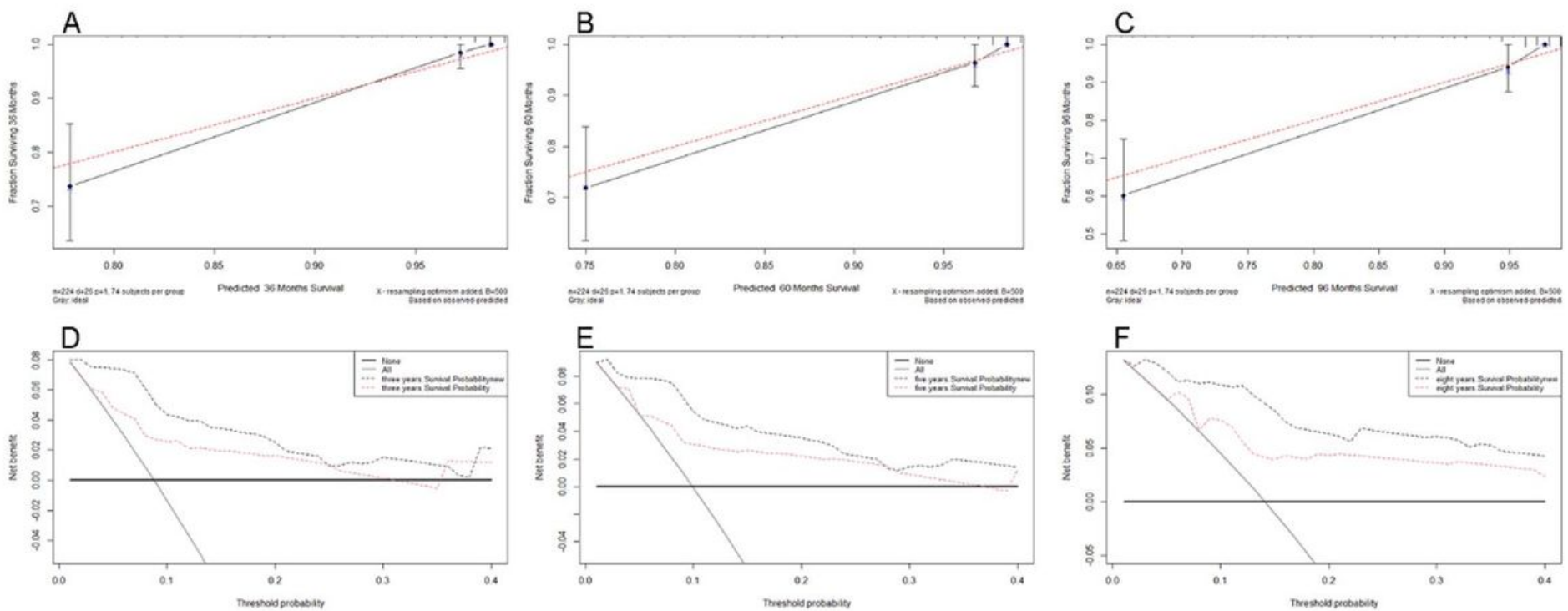

Figure 6 
The first row is the calibration of the nomograms using the validation dataset for 3-year (A), 5-year (B) and 8-year (C) overall survival (OS). The second row was decision curves of nomogram and traditional prediction method for 3-year (D), 5-year (E), and 8-year (F) OS. 